NYÍRI, Kristóf

\title{
INTRODUCING THE PAPERS IN THIS SECTION
}

With its unprecedented wealth of images, animations and videos, the digital-networked world poses a completely new challenge to the entire educational system, in particular, also, to higher education. In what sense can we speak of a visual liberation, a visual homecoming? How should visual contents be combined with traditional textual ones, to what extent are images autonomous carriers of meaning, what does a logic of images - still or moving - amount to, how can display-sized multimedia documents be structured, and integrated into a networked system? How can a conservative balance be created between the classical world of texts and the new world of images, what does the responsibility of intellectuals mean in an age of transient texts? At the same time how can pictorial communication alleviate the depersonalizing effects of digitally mediated connections?

It is in this context of issues and questions the Visual Learning Lab (VLL), at the Department of Technical Education, Budapest University of Technology and Economics, pursues its inquiries. On Nov. 14-15, 2014, the VLL held its 5th international conference: VISUAL LEARNING: PICTURES - PARABLES - PARADOXES. In the call for abstracts for the conference we have called attention to a particular set of problems bedevilling cutting-edge educational theory and practice at the very present juncture: "With the linguistic turn having become past history, and the iconic turn no doubt victorious, second thoughts are gradually arising: Is that victory not a much too complete one? Are philosophers, cognitive scientists, media theorists, and educationalists in particular, sufficiently aware of the dangers the neglect of a polished verbal logic and of verbal culture imply? Maintaining the right equilibrium between the pictorial and the verbal has once more become a burning issue." While this was, then, the main perspective we formulated, we listed, too, a much broader set of possible topics, such as: educational theory and visual learning; resemblance, representation, reality; image and language; images and media theory; visual rhetoric; pictorial meaning; pictorial communication; visual imagery; visual intelligence; the visual mind; the image problem in the history of philosophy; visual argument; scientific visualization; visualization and higher education; images in the network age.

The conference submissions underwent a blind peer-review process. Eventually, fortyfour researchers from twelwe countries presented their scientific results. The edited versions of some twenty of the talks will be published in the 5th volume of our VISUAL LeARnING series, under the title Beyond Words: Pictures, Parables, Paradoxes, to appear by September 2015. And four of the talks have been selected for publication in the present English issue of Opus \& Educatio.

The first of these, by András Benedek, sets the stage by sketching the history of the VLL project - a project he has been, let us say here, a main initiator and organizer of. Benedek then goes on to discuss that what has been our main issue at the conference, focussing on the intriguingly unstable ratio of the verbal and the pictorial when examining the gamut of successful, or inded unsuccessful, educational messages. A felicitious metaphor Benedek introduces - or is it more than a metaphor? - is that of the mathematical notion of the parabola, a metaphor suggesting the endeavour of keeping equal distance from a point and a line, just as perhaps ancient parables strove to keep equal distance between points of views expressed in an image on the one hand, and textual lines inspired by those points of 
views on the other. At one stage, Benedek uses another metaphor - or is it more than a metaphor? - to refer to the intertwining of the pictorial and the verbal: the metaphor of comics. And precisely comics is the explicit and central theme of the highly interesting paper by Krisztina Szabó. She argues that "reading digital texts containing visual elements is a nonlinear kind of reading which is indeed very much like comic reading". Szabó stresses that "visual elements which appear on digital interfaces have strong effects and influence on our cognitive processes, especially on reading and text comprehension". In pursuing her argument, Szabó spells out in detail what the notion of a "visual element" actually covers. She considers visual elements to be "pictures, graphics, and any kind of design elements of a website, word clouds and gifs. The interactive advertisements and the graphical elements of a Prezi or a Power Point presentation are also visual elements". Prezi, a popular online presentation tool with essentially Hungarian roots, is the topic of Anna Szlávi's paper "Metaphors Matter: The Analysis of Prezi in Search of Visual-Verbal Metaphors". There is, as Szlávi shows in her fascinating analysis, more to Prezi than meets the eye. Prezi's founding philosophy is clearly not independent of cognitive science in general, and conceptual metaphor theory in particular. However, Prezi's users are quite generally entirely unaware of, and certainly do not exploit, this dependence. Similarly unaware are, as Kristóf Fenyvesi and Raine Koskimaa explain in their paper, the users of some very specific digital games of the complicated metaphysics underlying their playful, but often also painful, employment of "impossible figures", figures optically well-structured, with a design however actually not yielding to be logically figured out. Fenyvesi, Koskimaa, and their associates have been for quite some time now conducting impressive experiments showing how dealing with, trying to understand, illusive images is conducive to mathematics education. Mathematics is, for most people, difficult; "impossible figures" become, to any onlooker, difficult on second look; the two fields, appropriately joined, might become a joyous playing field.

Visual learning might have been seen to be an easy playing field until recently, even if regarded with suspicion from the point of view of mainstream educational theory. The suspicion is still there, we feel; but the playing field has become an actually more difficult, because better understood, domain for innovative pedagogy. Game over? On the contrary, it just begins. 